\title{
PARADIGMAS DEL DERECHO, REFLEXIÓN Y DERECHOS SOCIALES
}

\author{
Daniel Alejandro Dodds Berger* \\ Universidad de Chile, Chile \\ doddsdaniel@gmail.com
}

RESUMEN: Dentro del trabajo más reciente del profesor Jürgen Habermas se destacan sus trabajos en materia jurídica. En específico, su propuesta de un nuevo paradigma del derecho que supere las deficiencias de los modelos formal-burgués y del Estado social. A partir del concepto de paradigma del derecho y de una exposición del desenvolvimiento de estos dos paradigmas y de sus consecuencias, se puede entender el desarrollo evolutivo de los sistemas jurídicos occidentales, como también la contradicción que hoy enfrentan. El paradigma procedimental del derecho y, en relación con este, la propuesta del profesor Gunther Teubner de un derecho reflexivo, será el centro del siguiente trabajo, realizado como una búsqueda de respuestas a estas contradicciones. La reflexión, como punto central de estos desarrollos teóricos, servirá para integrar la problemática de los derechos sociales. Respecto a los cuales, se dará también una breve y provisional aproximación respecto a su situación en los países subdesarrollados, específicamente, en los países latinoamericanos.

Palabras clave: paradigma, procedimentalización, reflexión, derechos sociales.

ABSTRACT: In the recent work of Professor Jürgen Habermas highlights its work on legal matters. Specifically, the proposal of a new paradigm of law to overcome the deficiencies of formal law and social state law. Using the concept of paradigm of law and an exhibition of the development of these two paradigms and their consequences, one can understand the evolutionary development of Western legal systems, as well as the contradiction faced today. The procedural paradigm of law and, in connection with this, the proposal of Professor Gunther Teubner a reflexive

* Universidad de Chile - Facultad de Derecho. Egresado, doddsdaniel@gmail.com, Teléfonos: 2834628/ 92128957. Ayudante de Derecho Constitucional e Introducción al Derecho - Facultad de Derecho de la Universidad de Chile. 
law, will be the focus of this paper, conducted a search for answers to these contradictions. Reflection, as a central point of these theoretical developments, will serve to integrate the issue of social rights. For which, there also a brief and tentative approach regarding the situation in underdeveloped countries, specifically in Latin America.

Keywords: paradigm, proceduralization, reflection, social rights.

\section{INTRODUCCIÓN}

Los procesos de transformación del derecho en relación con la sociedad, en gran medida no han sido del interés de los juristas. Ya sea por una cuestión metodológica o por una decisión teórica, el derecho ha venido siendo estudiado de forma independiente a los procesos de cambio que se han producido en las sociedades occidentales. Esto ha originado que muchas transformaciones jurídicas, más que como cambios, hayan sido vistas como una crisis del derecho. Una crisis de lo que es el derecho.

Las perspectivas evolutivas del derecho pretenden comprender la transformación del sistema jurídico en relación con los cambios que se han producido en las respectivas sociedades. Así, el derecho pierde su carácter estático y se hace reflexiva la cuestión de cómo se estructura. Al final, queda en claro el carácter político que hay detrás de ello.

El estudio de Jürgen Habermas sobre la evolución del derecho y de los paradigmas que se han desarrollado a través de esta, tiene el objetivo de demostrar cómo la lógica, la reflexión y las formas jurídicas no tienen un carácter perpetuo, sino que se han ido desarrollando a la par de los cambios desarrollados en las sociedades occidentales, desde el advenimiento de la modernidad.

Lo que demuestra también esta aproximación al derecho, es cómo los diferentes modelos de sociedad que han imperado en un sistema jurídico han determinado su desarrollo y sus limitaciones. Por lo que frente a las contradicciones a que nos enfrentamos hoy en nuestros sistemas jurídicos, una nueva lectura parece ser necesaria.

Este trabajo comenzará por el estudio del concepto de paradigma del derecho y su utilidad en el estudio de los sistemas jurídicos (1); posteriormente se examinará el desarrollo del paradigma formal-burgués y del Estado social (2). En tercer lugar, expondré lo que Habermas ha llamado, siguiendo a Rudolf Wiethölter, la procedimentalización del derecho o paradigma procedimental, como además la propuesta del profesor Gunther Teubner sobre el desarrollo de un derecho reflexivo (3). Por último, haré un breve comentario sobre las implicancias que tiene este nuevo paradigma para los derechos sociales, en especial, en los países latinoamericanos (4). 


\section{PARADIGMAS DEL DERECHO}

En general cuando hablamos de paradigmas nos estamos refiriendo a un modelo o ejemplo. En el ámbito científico un paradigma refiere a un modelo epistemológico, el cual provee de un contexto para el desarrollo de las diferentes teorías y explicaciones, a través de una serie de directrices. En las ciencias sociales, que es lo que nos interesa, un paradigma puede ser resumido como "una forma de diagnóstico, basada en una reconstrucción racional de formas de consciencia, filtradas y sintetizadas en una teoría, que busca orientar la acción"1.

En el campo del derecho la idea de paradigma como un modelo, se puede inferir de cuando diferentes teóricos, juristas o usuarios del sistema jurídico hacen referencia a una cierta visión social o modelo social observable en este. Con esto se entiende que el derecho tendría una imagen de cómo es la sociedad. Esta visión inscrita en el sistema jurídico, refiere “a esas imágenes implícitas sobre una sociedad que guían la práctica actual de producir y aplicar el derecho" 2 . Esta comprensión de fondo se puede inferir, en primer término, de las decisiones de los tribunales de justicia, ya sea en términos dogmáticos o histórico-jurídicos, sin embargo, esta labor de experto se vuelve insuficiente. Desde esta óptica, se puede caer fácilmente en un estudio sobre las representaciones que tienen los jueces sobre la creación de normas jurídicas. Por lo tanto, se hace menester integrar al punto de vista de los tribunales el de los sujetos en general, es decir, el de la cultura jurídica. Así, "los paradigmas jurídicos, mientras funcionan en forma de un saber atemático de fondo, se apoderan de la conciencia de todos los actores, de la conciencia de los ciudadanos y de los clientes, no menos que de la del legislador, la Justicia y la Administración"3.

Los cambios sociales producen modificaciones en las estructuras jurídicas y en la cultura jurídica, es decir, se puede sostener una covariación social y jurídica. Cuestión a la que se llega al considerar al derecho como parte de la sociedad. Entonces, en un sistema jurídico en diferentes épocas, existe una imagen de la sociedad de la cual forma parte, cuestión que busca hacer patente la compresión paradigmática del derecho. Esta describe los procesos de evolución que este ha enfrentado, tal como se han expresado en él mismo. Una reflexión en este sentido, es la que lleva a los actores a una idea sobre cómo se ha desarrollado y cómo debiera realizarse el contenido normativo del Estado de derecho, es decir, da cuenta del carácter político de la cuestión.

Un ejemplo de este carácter paradigmático, es posible de observar en el fenómeno llamado constitucionalización del derecho, o de forma más específica, constitucionalización

\footnotetext{
Arato, Andrew. Reflexive Law, Civil Society, and Negative Rights, en Cardozo Law Review, New York, N 17, 1996, p. 784. Habermas, Jürgen. Paradigms of Law, en Cardozo Law Review, New York, Nº 17, 1996, p. 770.

Habermas, Jürgen. Facticidad y Validez: Sobre el derecho y el Estado democrático de derecho en términos de teoría del discurso, 4a edición, Madrid, España, Editorial Trotta, 2001, p. 477.
} 
del derecho privado. El cambio de paradigma es percibido como una crisis del derecho, toda vez que el derecho de un Estado interventor conlleva una irradiación de los derechos fundamentales a todas las áreas, rompiéndose así la lógica formal del sistema. Desde la perspectiva de los paradigmas, estas variaciones, más que crisis, significan cambios, variaciones del derecho que no por eso destruyen su identidad como tal.

\section{DEL PARADIGMA DEL DERECHO FORMAL-BURGUÉS AL PARADIGMA DEL ESTADO SOCIAL}

Para Habermas los cambios de paradigmas en el derecho se pueden comprender desde la relación entre autonomía privada y autonomía pública. Partiendo desde esta distinción se han distinguido dos paradigmas:

\section{El PARADIGMA DEL DERECHO FORMAL-BURGUÉS}

El modelo social burgués partía de una clara separación entre Estado y Sociedad. A través de esta diferenciación se dejó atrás la sociedad feudal caracterizada por su estratificación y se asentaron las bases para el desarrollo de la autonomía individual. Esta trasformación conllevó que "los distintos ámbitos funcionales de la sociedad (fundamentalmente los económicos, pero no menos los culturales) fueran liberados del control político y entregados al mercado, bajo el cual pudieron desarrollarse mediante decisiones individuales de voluntad que seguían sus propios criterios de racionalidad" ${ }^{4}$. La sociedad civil era entendida como una esfera de ausencia de poder, que venía protegida y estructurada a través de derechos fundamentales de carácter negativo. En este modelo social, la sociedad quedaba encargada del bienestar y la justicia, quedando el Estado en una posición secundaria. A este le correspondían las funciones de defensa frente a las afectaciones de la libertad, que se dieran durante el libre despliegue de las fuerzas sociales.

En cuanto al derecho, el paradigma formal-burgués nos presenta una sociedad estructurada en términos de derecho de privado. Partiendo del supuesto de la autonomía de los sujetos, el derecho viene a delimitar y proteger esferas de libertad, en las cuales estos pueden perseguir sus intereses privados. El derecho establece condiciones en que puede ejercitarse la coerción, bajo las cuales "el arbitrio de uno pueda conectarse o conciliarse con el arbitrio del otro conforme a una ley general de libertad" 5 . Este principio general del derecho aseguraba una igual libertad para todos los sujetos, los cuales a través de su ejercicio regularían sus relaciones en una sociedad centrada en la economía. La legitimidad del derecho en este modelo venía asegurada por la dimensión semántica de la ley: esta debería ser general y abstracta.

4 Grimm, Dieter. Constitucionalismo y derechos fundamentales, 1a edición, Madrid, España, Editorial Trotta, 2006, p. 178.

5 Kant, Immanuel. Metafísica de las Costumbres, 2a edición, Madrid, España, Editorial Tecnos, 1994, p. 39. 
Este paradigma descansa en un formalismo jurídico que se sigue de la racionalidad formal del derecho mismo, la cual es desarrollada y protegida por expertos que sistematizan, en términos dogmáticos, el derecho como un todo lógico ${ }^{6}$. Un corpus de esta forma estructurado, permitía que el derecho fuera visto como un conjunto ordenado y predecible de normas, unitario y en donde la vinculación de la Justicia y la Administración a la ley, se traducían en seguridad jurídica. Cuestión fundamental, toda vez que "la seguridad jurídica, es decir, la vinculación de las funciones del Estado a normas generales, protege, junto a las libertades codificadas por el sistema de derecho privado burgués, el orden del mercado libre"7.

\section{El PARAdigma del DERECho del Estado SOCIAL}

El dogma de que a través de relaciones sociales cortadas en términos de derecho privado, podría generar espontáneamente justicia social, fue pronto puesto en duda por avezados $\operatorname{críticos}^{8}$. Las críticas al derecho formal burgués produjeron una ola de reformas que buscaban equiparar en situaciones concretas, las desigualdades que impedían que el derecho a una igual libertad se pudiera concretar. Quedaba de manifiesto que "el derecho de cada uno a poder hacer u omitir lo que quiera en el marco de la atenencia a las leyes, solo se cumple bajo la condición de que esas leyes garanticen la igualdad de trato en el sentido de igualdad jurídico-material" 9 . Así, sin cambiar las premisas, se desarrolló una nueva lectura del derecho, el cual implicaba una nueva imagen de la sociedad, un nuevo paradigma.

En una sociedad en que las funciones del Estado se extienden, el derecho se transforma, llegando a ámbitos de la sociedad a que antes no llegaba. Este proceso de jurificación introdujo elementos materiales a los sistemas jurídicos, sacudiendo la racionalidad de estos con principios de justicia.

Esta politización de la sociedad a través de la intervención del Estado produjo grandes cambios en las sociedades occidentales, equilibrando las relaciones sociales allí en donde existían situaciones de poder asimétricas, lo cual condujo a un gran bienestar e integración social, en aquellos países en que las condiciones apropiadas lo permitieron.

Sobre esto ver: Kelsen, Hans. Teoría Pura del Derecho, $2^{a}$ edición, Ciudad de México, México, Editorial UNAM, 1982, pp. 83-122.

7 Habermas, Jürgen. Historia y crítica de la Opinión Pública. La transformación estructural de la vida pública, $9^{a}$ edición, Barcelona, España, Editorial Gustavo Gili, 2006, p. 115.

8 Para estos quedaba claro que una igual libertad de todos los sujetos, no podía desarrollarse si el derecho era ciego a las diferencias materiales en que se encontraban estos. El desarrollo de los movimiento obreros de finales del siglo XIX y principios del siglo XX llevaron a que estas críticas se plasmaran en la cultura jurídica y posteriormente en los sistemas jurídicos. De esta forma, el derecho sufrió un proceso de transformación que llevó al advenimiento del llamado paradigma del derecho del Estado social.

9 Habermas, Jürgen. Facticidady Validez, cit., p. 484. 
Pese a los avances a que condujo este nuevo modelo, comenzaron a aparecer críticos de izquierdas y derechas, que hacían ver los problemas que aparejaba esta materialización del derecho. Estas críticas se pueden resumir en dos:

a. Materialización como crisis de la racionalidad del derecho: los defensores de la racionalidad formal del derecho interpretaron este cambio como una crisis. Para Weber, la racionalidad formal que caracteriza al derecho positivo "significaba calculabilidad e indiferencia ante todo presupuesto valorativo o ante todo fin ético o material" ${ }^{10}$. La racionalidad formal de un sistema legal se puede observar en el respeto de una serie de condiciones. Gunther Teubner las sintetiza de esta forma: un sistema legal racional formalmente crea y aplica un cuerpo de reglas universales, y la racionalidad formal del derecho se basa en un cuerpo de profesionales del derecho que emplean un peculiar racionamiento legal para resolver conflictos específicos ${ }^{11}$.

Un ordenamiento jurídico que incluyese un contenido material a través de un conjunto de principios, plasmados en el nivel más alto en principios de carácter constitucional, perdería de esta forma su sistematicidad y coherencia lógica, perdiendo esa calculabilidad que tanto destaca Weber, abriéndose a la arbitrariedad y al particularismo.

b. Materialización como causa de un déficit de legitimidad: el modelo del Estado social produciría un problema en términos de legitimidad toda vez que rompía con las características semánticas del derecho formal burgués, que aseguraban protección a los individuos. El derecho ya no se legitimaba en la forma clásica de la ley, es decir, "la dominación ejercida en tales formas por la sola razón de que semejante dominación cumpla determinados requisitos funcionales para la persecución privado-autónoma y la persecución racional con arreglo a fines de los propios intereses de cada uno" ${ }^{2}$. La materialización del derecho a través de los programas de un Estado cada vez más preocupado de aspectos de integración, con carácter finalista, produciría un debilitamiento de la protección al individuo, al remover las barreras para la intervención burocrática en diferentes aspectos de interacción humana (escuela, familia, trabajo, etc.).

10 Fariñas, María José. La sociología del derecho de Max Weber, 1a edición, Editorial Civitas, Madrid, España, p. 278.

11 Teubner, Gunther. Substantive and reflexive elements in modern law, en Law and Society Review, New Jersey, volumen 17, número 2, 1983, p. 240.

12 Habermas, Jürgen. Derecho y Moral, en Complementos y Estudios Previos, En su: Facticidad y Validez: Sobre el derecho y el Estado democrático de derecho en términos de teoría del discurso, 4a edición, Madrid, España, Editorial Trotta, 2001, p. 541. 


\section{PROCEDIMENTALIZACIÓN DE LA CATEGORÍA DEL DERECHO Y REFLEXIÓN}

El paradigma del Estado social a través de una serie de reformas, pero sin renunciar a las mismas premisas, intentó corregir los costos sociales que el modelo burgués traía consigo, utilizando la intervención del Estado para estos efectos. Sin duda, en donde se desarrollaron las estructuras necesarias para su implementación este modelo trajo grandes beneficios. Sin embargo, al transcurrir los ańos comenzaron a verse los problemas que atraía aparejado y que ponían en peligro el propio fundamento que había dado lugar a su nacimiento: el asegurar la autonomía de los sujetos.

Frente a los problemas que presenta el modelo social se ha propuesto una vuelta a una concepción liberal del derecho, fundada en derechos de carácter negativo ${ }^{13}$. Opción que nos parece inviable dado los grandes costos sociales que esto puede conllevar. La alternativa, para Habermas, sería una radicalización en términos democráticos del derecho a través de un nuevo paradigma: el paradigma procedimental.

Para proponer este nuevo paradigma, Habermas parte de tres premisas: (1) El retorno a la sociedad civil y a su derecho, tal como propugnan los neoliberales, está vedado; (2) la consigna del redescubrimiento del individuo viene provocada por un tipo de juridificación ligada al Estado social que amenaza con convertir en lo contrario que es su objetivo declarado, es decir, el restablecimiento de la autonomía privada; (3) el proyecto del Estado social ni simplemente hay que ratificarse en él, ni tampoco se lo puede interrumpir ${ }^{14}$, sino que debe proseguir en un plano superior de reflexión ${ }^{15}$. La procedimentalización de la categoría del derecho, entonces, vendría a ser una respuesta orientada a disminuir la tensión entre lo colectivo y lo individual, entre estructura y sujeto, entre sistema y mundo de la vida.

El análisis de Habermas sobre este punto parte de la interna conexión entre autonomía privada y autonomia pública, las cuales se remiten una a la otra. Esto significa que el ejercicio de la autonomía pública o ciudadana es necesaria para el resguardo y desarrollo de la autonomía privada, y viceversa, el ejercicio de las libertades individuales es presupuesto de una formación de la voluntad y opinión política, que pueda llamarse libre y racional.

Esta exigente propuesta requiere un desarrollo del derecho acorde a los nuevos retos que enfrentan las sociedades complejas, resultado de la evolución antes estudiada. Habermas sostiene que no existe una forma jurídica determinada que pueda ser característica del emergente paradigma procedimental. Los dos modelos anteriormente estudiados, privilegiaron

13 Para una propuesta de izquierdas sobre este punto, ver: GRImm, Dieter. ¿¿Retorno a la comprensión liberal de los derechos fundamentales? En su: Constitucionalismo y derechos fundamentales, 1a edición, Madrid, España, Editorial Trotta, 2006, pp. 155-173.

14 Sobre esto, ver: Habermas, Jürgen. Escritos Políticos, 3a edición, Barcelona, España, Editorial Península, 1997, pp. 113-134.

15 Habermas, Jürgen. Paradigms of Law, cit., pp., 777-778. Cf. también Habermas, Jürgen. Facticidad y Validez, cit., p. 492. 
determinadas formas de derecho, el derecho formal y el material, respectivamente, pero no parece existir razones para privilegiar un tipo sobre otro, sino que la decisión vendría a ser el resultado de una reflexión sobre la forma de derecho que permita "asegurar uno actu, la autonomía privada y la autonomía pública de los ciudadanos, por vía de que todo acto jurídico pueda entenderse a la vez como una contribución a la configuración política autónoma de los derechos fundamentales, es decir, pueda entenderse como elementos de un proceso constituyente planteado a largo plazo" 16 .

Así las cosas, el paradigma procedimental sugiere una reflexión en torno a la justificación y aplicación del derecho que sea acorde con el haberse hecho evidente cómo las estructuras jurídicas, en su relación con las estructuras sociales, definen el desarrollo del ordenamiento jurídico. Es decir, supone una visión menos ingenua y más atenta, que supere la simplicidad del derecho liberal, pensado en términos de relaciones entre sujetos, y el paternalismo del Estado social, consecuencia del haberse hecho notoria la necesidad de planificar y regular las interacciones entre estructuras y sujetos, con el fin de asegurar esta autonomía de los sujetos. El paradigma procedimental, entonces, a nivel de fundamentación, debiera hacer visible los problemas a que se enfrenta la producción del derecho ante los imperativos sistémicos de un sistema económico avasallador, y de un sistema político que tiende a autonomizarse en términos plebiscitarios. Busca, en palabras de Rudolf Wiethölter, "no tanto un desarrollo de garantías de rendimiento social (como "derecho de libertad"), o concesiones (como políticas administrativas), pero sí la posibilidad (y la organizaciones, procedimientos y otras cuestiones para su realización) de tales garantías y concesiones a través de arreglos conducidos legalmente" 17 .

Pese a que no se señale ningún tipo de derecho como característico de este paradigma procedimental, ya se han desarrollado propuesta en torno a un emergente derecho que encarna algunas de las premisas de esta propuesta: el derecho reflexivo.

Tal como es expuesto por Teubner, este derecho viene a ser el resultado de la transición desde las sociedades del bienestar a las actuales. El derecho reflexivo es una alternativa al proceso de reformalización que es posible observar actualmente, pero conservando similitudes con el derecho materializado como es su orientación a intervenir en procesos sociales. Para examinar los distintos tipos de racionalidad del derecho, Teubner divide las cosas en tres niveles ${ }^{18}$. Como ya hemos avanzado en lo que se refiere a la racionalidad formal y a la material, en este punto solo desarrollaremos lo referido a la llamada racionalidad reflexiva:

16 Habermas, Jürgen. Facticidad y Validez, cit., p, 493.

17 Wiethölter, Rudolf. Proceduralization of the Category of Law, En: Critical Legal Thought. An American-German Debate, ed. por Ch. Joerges and D. M. Trubek, 1a edición, Baden-Baden, Alemania, Editorial Nomos, 1989, p. 510.

18 Teubner, Gunther. Substantive and reflexive elements in modern law, cit., pp. 254-257. 
1) Justificación del derecho (racionalidad de las normas): la justificación de las normas no vendrá determinada por la autonomía privada, ni por la regulación del comportamiento. El derecho reflexivo persigue el desarrollo de una autonomía regulada, busca una regulación sistémica a través de normas de organización y procedimientos.

2) Funciones externas del derecho (racionalidad sistémica): en este nivel el derecho reflexivo busca estructurar y reestructurar ámbito en donde se puedan desarrollar distintos tipos de discursos (procedimientos), con lo que se pueda salvar la integridad del sistema jurídico y la lógica propia de cada uno de estos discursos.

3) Estructura interna del derecho (racionalidad interna): El derecho reflexivo "depende de normas procesales que regulen procesos, organizaciones, y que distribuyan derechos y competencias (...) el control legal de las acciones sociales es indirecto y abstracto, debido a que el sistema solo determina las premisas organizativas y procesales de una futura acción"19.

A través del derecho reflexivo, entonces, ya no se busca determinar directamente soluciones para casos particulares, o determinar fines concretos por parte del legislador; el objetivo, más bien, es capacitar a los sujetos para que puedan regular por su propia cuenta sus asuntos. Un ejemplo de esta forma jurídica la podemos observar en el derecho laboral, concretamente en materia de negociación colectiva.

\section{EL PARADIGMA PROCEDIMENTAL Y LOS DERECHOS SOCIALES EN LATINOAMÉRICA}

El paradigma procedimental del derecho pretende superar la ceguera del derecho formal burgués y el paternalismo del derecho del Estado social: "este tipo de derecho no tiende tanto a ofrecer prestaciones sociales, y tampoco garantías jurídicas, cuanto a establecer las condiciones de posibilidad de tales prestaciones y garantías mediante estructuras procesales" 20 .

En relación a los derechos sociales, un derecho procedimental podría establecer las condiciones para el desarrollo de un derecho orientado a la deliberación. El problema que ha traído la materialización del derecho es el de la imposición de un derecho utilizado de manera instrumental. Respecto a esto, el derecho procedimental es una determinación solo parcial del derecho desde el punto de vista de la tarea legislativa, siendo el output del siste-

19 Ibid., p. 255.

20 La Torre, Massimo. Derecho y conceptos de derecho. Tendencias evolutivas desde una perspectiva europea, en Revista del Centro de Estudios Constitucionales, Madrid, Espańa, volumen No 16, 1993, p. 88. 
ma, en un caso particular, el resultado de la deliberación de los sujetos bajo las condiciones procedimentales antes estructuradas. El derecho procedimental permitiría llegar a soluciones más acordes a las expectativas de los sujetos y menos determinadas de forma imperativa por el legislador o la administración.

Sobre el papel que pueda jugar una reflexión a nivel superior acerca de las fundamentación de las normas en materia de derechos sociales, todavía queda por verse. Más aún en los países subdesarrollados, específicamente en los países latinoamericanos. Aquí el problema se vuelve más agudo todavía, ya que la dimensión simbólica del derecho, y en especial de la Constitución, se viene acrecentado. El déficit de regulación que se observan en los países europeos, en parte como efecto de la ampliación de funciones del Estado y de la aparición de nuevos actores cuasipolíticos, en Latinoamérica se extrema.

En las sociedades subdesarrolladas el derecho, debido a su función, se ve forzado a aplicar normas que se encuentran cuasiinstitucionalizadas constitucionalmente o establecidas de forma legal, pero que no han sido vinculantes efectivamente para la política (o la administración). En este sentido, las decisiones de los tribunales en casos de derechos sociales deben enfrentarse a situaciones de gran ambivalencia en cuanto a los factores a determinar en un caso concreto, pero además, se ven enfrentados a tener que, desde el punto de vista del sistema político, reprocharle su falta de concreción respecto al desarrollo de estos derechos. Así, las decisiones de los tribunales, en nuestro contexto, tienen limitadas posibilidades de concretarse tal y como podrían venir exigidas en condiciones adecuadas.

Esta característica del desarrollo de los derechos sociales en Latinoamérica, permite sostener que muchas de estas decisiones presentan un carácter simbólico desde el punto de vista objetivo de los derechos: "Lo simbólico implica un lenguaje o discurso en el que hay un desplazamiento de sentido hacia otra esfera de significaciones (...) El actuar simbólico es connotativo en la medida en que adquiere un sentido mediato e impreciso, que se ańade a su significado inmediato y manifiesto" 21 Este desplazamiento a la perspectiva de la política vendrá a ser una especie de "recordatorio" respecto al carácter normativo que tienen los derechos sociales. Las decisiones de los tribunales vendrían a irritar a un sistema político que tiendan a independizarse y a enfrentar el derecho en forma instrumental.

Por último, el desarrollo del derecho procedimental en los países latinoamericanos, podría ser útil para desarrollar una reflexión acerca de la importancia de los derechos sociales para la estabilidad de toda sociedad moderna. Ayudaría a un desarrollo progresivo de estos, pero con la participación de los propios afectados, teniendo en cuenta el marco institucional de cada sociedad, es decir, permitiendo contextualizar de alguna forma la discusión.

21 Neves, Marcelo. La fuerza simbólica de los derechos humanos, Revista Doxa, Cuadernos de Filosofía del Derecho № 27 , 2004, p. 147. 


\section{CONCLUSIÓN}

Hasta aquí hemos desarrollado una visión paradigmática del derecho, que se enmarca en una teoría evolutiva de este. La opción que tomamos fue exponer los dos paradigmas que han sido analizados comúnmente, para luego desarrollar la propuesta de Habermas sobre un nuevo paradigma del derecho. Este paradigma pretende llevar el desarrollo del derecho a un nivel más reflexivo, que puede hacer justicia a la incipiente trasformación del derecho desde el punto de vista procedimental, con lo cual poder enfrentar la tensión presente en los sistemas jurídicos contemporáneos.

La propuesta de Habermas (y en parte también de Teubner) traza un camino de estudio para enfrentar los cambios que se están produciendo en el derecho actualmente, y da una salida al retroceso que significa la reformalización del derecho. Sin embargo, existen algunos aspectos que debemos someter a crítica:

1) Si bien la teoría de Habermas sobre la procedimentalización del derecho se presenta como una propuesta compleja tal y como es la realidad de las sociedades a las que se refiere, falta todavía un mayor desarrollo a nivel empírico ${ }^{22}$. Los niveles de análisis normativo y empírico aparecen en ocasiones mezclados, lo que produce una superposición que no permite sacar conclusiones prácticas. Cuestión, por supuesto, que no derrumba el edificio, pero sí hace necesario su complemento.

2) Habermas argumenta que el paradigma procedimental no privilegia una forma de derecho en especial. De hecho, las referencias al derecho reflexivo no son abundantes en su obra, lo cual hace depender la aplicación de una u otra forma de derecho de la reflexión a través de un proceso deliberativo. Sin embargo, el paso de un paradigma a otro, es decir, la evolución del derecho, hace pensar que podríamos estar ante el desarrollo de nuevas formas de derecho, cuestión que no aparece en su trabajo.

3) La propuesta procedimental y el derecho reflexivo, no son iguales, pese a las similitudes, existen diferencias que podrían explicar el límite trazado para aplicar este último dentro del modelo propuesto por Habermas. El problema que es posible vislumbrar en el derecho reflexivo tiene que ver con el desarrollo corporativista que podría presentar. Tal como lo manifiesta Teubner, el derecho reflexivo vienen a llenar los problemas de integración entre subsistemas sociales que se han diferenciado estableciendo espacios de deliberación, estructurados jurídicamente, en donde sea posible resolver conflictos de forma responsable. Sin embargo, la primacía de una deliberación de

22 Esto ya se le ha criticado a la teoría jurídica de Habermas en conjunto. Ver: Peters, Bernhard. On reconstructive legal and political theory, En: Habermas, Modernity and Law, Nueva, ed. Deflem, Mathieu 1a edición, London, Reino Unido, editorial Sage Publications Ltd., 1996, pp. 101-134. 
ciudadanos respecto a la fundamentación de normas no viene garantizada en este modelo, sino que, al asumir una perspectiva sistémica (organizacional), serán más bien organizaciones las que participarán en la solución de los conflictos.

4) En relación al desarrollo del derecho en los países subdesarrollados, la teoría de Habermas ha sido construida teniendo en vista a la ordenada sociedad alemana. Si bien esto puede ser matizado, no deja de existir una deuda en esta propuesta sobre el desarrollo del derecho en los países de la modernidad periférica. 


\section{REFERENCIAS BIBLIOGRÁFICAS}

Arato, Andrew. Reflexive Law, Civil Society, and Negative Rights, en: Cardozo Law Review, New York, $\mathrm{N}^{\circ} 17$, 1996.

Fariñas, María José. La sociología del derecho de Max Weber. Madrid, España: Editorial Civitas, 1999.

Grimm, Dieter. Constitucionalismo y derechos fundamentales. Madrid, España: Editorial Trotta, 2006.

Habermas, Jürgen. Derecho y Moral, en Complementos y Estudios Previos, En su: Facticidad y Validez: Sobre el derecho y el Estado democrático de derecho en términos de teoría del discurso. -4a edición- Madrid, España: Editorial Trotta, 2001.

. Escritos Politicos. -3a edición- Barcelona, España: Editorial Península, 1997.

Facticidad y Validez: Sobre el derecho y el Estado democrático de derecho en términos de teoría del discurso. -4a edición-Madrid, España: Editorial Trotta, 2001.

. Historia y crítica de la Opinión Pública. La transformación estructural de la vida pública. -9a edición- Barcelona, España: Editorial Gustavo Gili, 2006. . Paradigms of Law, en: Cardozo Law Review, New York, Nº 17, 1996.

Kant, Immanuel. Metafísica de las Costumbres. -2a edición- Madrid, España: Editorial Tecnos, 1994.

Kelsen, Hans. Teoría Pura del Derecho. -2a edición- Ciudad de México, México: Editorial UNAM, 1982.

La Torre, Massimo. Derecho y conceptos de derecho. Tendencias evolutivas desde una perspectiva europea, en: Revista del Centro de Estudios Constitucionales, Madrid, España, volumen Nº 16, 1993.

Neves, Marcelo. La fuerza simbólica de los derechos humanos, en: Revista Doxa, Cuadernos de Filosofía del Derecho $\mathrm{N}^{\circ} 27,2004$.

Peters, Bernhard. On reconstructive legal and political theory, En: Habermas, Modernity and Law, Nueva, ed. Mathieu Deflem, London, Reino Unido, editorial Sage Publications Ltd, 1996.

Teubner, Gunther. Substantive and reflexive elements in modern law, en: Law and Society Review, New Jersey, Volumen 17, número 2, 1983.

Wiethölter, Rudolf. Proceduralization of The Category of Law, En: Critical Legal Thought. An AmericanGerman Debate, ed. por Ch. Joerges and D. M. Trubek, 1a edición, Baden-Baden, Alemania, Editorial Nomos, 1989. 